Relations industrielles

Industrial Relations

\title{
Du bon usage de l'Étude Économique dans l'entreprise, par J. Lesourne, Dunod, Paris, 1966, 173 pages.
}

\section{Paul Bouchard}

Volume 22, numéro 2, 1967

URI : https://id.erudit.org/iderudit/027797ar

DOI : https://doi.org/10.7202/027797ar

Aller au sommaire du numéro

Éditeur(s)

Département des relations industrielles de l'Université Laval

ISSN

0034-379X (imprimé)

1703-8138 (numérique)

Découvrir la revue

Citer ce compte rendu

Bouchard, P. (1967). Compte rendu de [Du bon usage de l'Étude Économique dans l'entreprise, par J. Lesourne, Dunod, Paris, 1966, 173 pages.] Relations industrielles / Industrial Relations, 22(2), 305-305.

https://doi.org/10.7202/027797ar

Tous droits réservés (C) Département des relations industrielles de l'Université Laval, 1967
Ce document est protégé par la loi sur le droit d'auteur. L'utilisation des services d'Érudit (y compris la reproduction) est assujettie à sa politique d'utilisation que vous pouvez consulter en ligne.

https://apropos.erudit.org/fr/usagers/politique-dutilisation/ 
de compréhension des éditeurs vis-ò-vis la position de l'auteur et vis-à-vis le syndicalisme américain. Nous considérons aussi comme déplorable, l'absence des notes et références de l'édition originale. En effet, si un lecteur sérieux veut refaire le même travail que $M$. Widick, mais en supposant une cutre conception du syndicalisme, l'édition fronçaise, tronquée de ces instruments ne lui sera que d'un maigre recours. Or, la conclusion que nous tirons de la lecture de cet ouvrage est que le point de vue de l'auteur est valable en tant que point de vue. La lecture de cette publication apporte beaucoup au lecteur. Elle devrait apporter, entre outres, le goût d'en savoir plus sur le syndicalisme américain et surtout de connaître d'outres points de vue.

\section{André PETIT}

\section{Du bon usage de l'Etude Economique dans I'entreprise, par J. Lesourne, Dunod, Paris, 1966, 173 pages.}

Comme son titre l'indique, l'objet de cet ouvrage est de traiter de l'utilisation des études économiques dans la gestion des entreprises. La généralité même de cet objectif suggère la variété des problèmes qui peuvent faire l'objet d'études économiques. Elles sont devenues à la fois nécessaires et possibles, parce qu'elles sont l'un des ospects de la construction intensive, par les générations présentes, d'une société économiquement très différenciée, dominée par la recherche technique et par le désir de maîtriser, grâce à la pensée scientifique, tous les processus de décision.

Au delà de cette approche générale apparaissent, d'une façon significative, certaınes données prépondérantes. Parmi celles-Ci notons que l'étude économique semble être l'une des nombreuses filles de la complexité des décisions de l'entreprise, qu'elle est en quelque sorte conditionnée par les attitudes des dirigeants d'entreprises et qu'elle s'intercale dans un processus qui commence par la prise de conscience d'un problème de décision ou du choix d'une politique qui se continue par la fixation d'un programme d'études ayant pour but d'éclairer le choix et qui se termine par une décision utilisant les résultats de l'étude. Cette dernière phrase, d'ailleurs, laisse supposer une dernière donnée qui s'inscrit comme fondamentale et qui se situe ou niveou de la collaboration entre les dirigeants d'entreprises et les hommes de recherche.
Les affirmotions ci-hout mentionnées montrent que l'utilisation des études économiques est un art qui n'est point si facile. Pour le maîtriser, l'auteur laisse entendre qu'il faut comprendre l'esprit des méthodes sons s'attacher à la lettre ni aux détails techniques. C'est pourquoi l'auteur consacre une partie de son ouvrage à l'étude de la technique économique appliquée à l'entreprise. Puis, dans une troisième partie l'auteur trace le panorama général des décisions de l'entreprise et des études qui peuvent les éclairer. Ce chapitre commande luimême les trois chopitres qui lui succèdent et qui sont plus particulièrement consacrés à la manière de poser trois séries de problèmes essentiels à l'entreprise: ceux qui concernent la politique commerciale, ceux qui concernent la politique d'utilisation des moyens de production et ceux qui concerrient la politique d'investissement.

Enfin, comme tout volume se termine par une conclusion, la dernière partie traite de l'avenir des études économiques dans l'entreprise à partir d'une analyse de la logique de l'évolution des techniques utilisées et des problèmes abordés, d'un intérêt à la gestion de l'entreprise et d'une recherche concomitante de l'ensemble des transformations probables, replaçant ainsi les études économiques dans le contexte plus vaste du déterminisme des modifications de la gestion industrielle.

\section{Paul BOUCHARD}

Principes de direction des entreprises, par Neely D. Gardner, version française de Michel de Jihef, Editions Gamma, Tournai, (Belgique), 3 volumes, 365 pages.

Principes de direction des entreprises est un cours en enseignement programmé (Turtortext). Dès la première page, on peut lire ceci: "Ce livre vous posera sans cesse des questions et corrigera vos erreurs en même temps qu'il vous fournira toutes les connaissances nécessaires ». On veut enseigner, fixer des connaissances précises et bien définies. Cet enseignement programmé se fait par questions et réponses vraies ou fausses. Une vérification continuelle de la compréhension est alors possible. Le cours vise une véritable formation pour le lecteur, c'est-à-dire l'amener à se transformer et à se juger.

Le premier volume traite d'abord de la perte de temps dans l'entreprise, et des diverses façons de l'éviter. Les principaux points abordés sont les interruptions, les 